\title{
The importance of adequate data pre-processing in early diagnosis: classification of arrhythmias, a case study
}

\author{
A. Rabasa ${ }^{1}$, A. F. Compañ ${ }^{2}$, J. J. Rodríguez-Sala ${ }^{1} \&$ L. Noguera ${ }^{1}$ \\ ${ }^{1}$ Operations Research Center, Universidad Miguel Hernández de Elche, \\ Spain \\ ${ }^{2}$ Department of Surgery and Pathology, Universidad Miguel Hernández \\ de Elche, Spain
}

\begin{abstract}
Data management can become very complex in the context of forecasting medical problems. Data collection, storage and analysis require the highest level of accuracy possible. The successful application of data mining techniques for the early diagnosis of disease or dysfunctions is increasingly more frequent among the scientific communities. However, as in any analytical method, the precision and reliability of the models provided by these techniques is absolutely dependent on the input data. If the quality of these data is not sufficient, the final accuracy can be greatly reduced to the point that the system becomes somewhat unproductive. This paper describes the main problems and how they can be properly solved at the pre-processing stage. Some of issues addressed are, for example: the detection of missing values (due to incomplete records), identification of outliers (often due to errors in measuring or recording devices), and discretization of numerical variables (where the context allows or suggests trying numeric values as nominal segments). Considering a public data base for arrhythmia from the UCI Repository, this study uses free Data Mining software to parameterize and run forecasting models and execute several computational experiments that show how the accuracy of predictions vary according to how you implement the critical pre-processing stage. The paper concludes providing a generic procedure that aims to apply the pre-processing of data in a methodical way and depending on the problems presented by the input data, and how it should be integrated into a global process of data management.

Keywords: Data Mining, pre-processing, forecasting, medicine, arrhythmia.
\end{abstract}




\section{Introduction}

\subsection{Pre-processing basic concepts}

The pre-processing stage is usually considered (Chen [1], Berry and Linnoff [2]) as a part of the general Data Mining schema (Figure 1), where, after the problem definition and data collection stages and prior to the model selection, the input data must be prepared to be adequately processed, at least from a formal and theoretical approach. However, in practice, basic pre-processing is implemented independently from the Data Mining model, while other pre-processing tasks are developed depending on it.

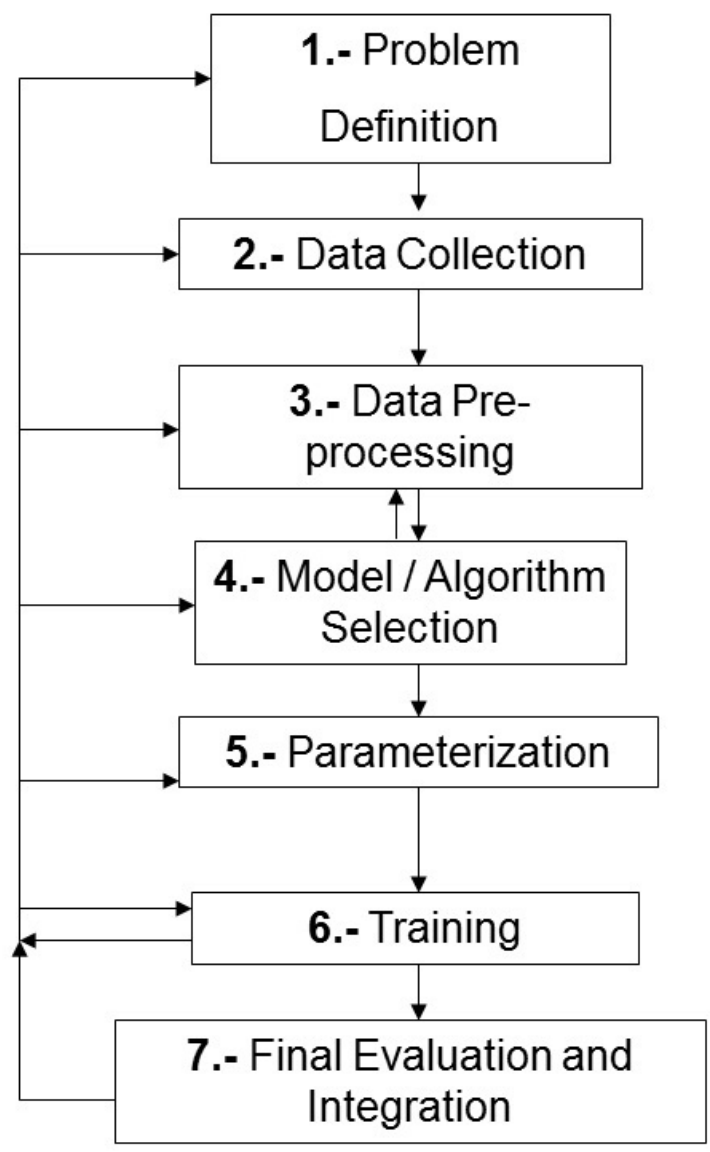

Figure 1: $\quad$ Pre-processing stages as a part of the Data Mining life cycle. 
The most common pre-processing tasks are as follows: for example, outlier detection (and maybe the outlier's replacement), missing value detection (and maybe missing value completion), filtering attributes (statistically insignificant or high error ratio), filtering uncompleted records (with missing high ratio values), and discretization of numerical values.

Pre-processing becomes necessary in different contexts, for example, where different sources are joined, where noisy real time data must be processed, or even when final accuracy must be improved. This paper focuses on the last scenario.

\subsection{Objectives}

This paper presents two main objectives:

- To demonstrate how different data pre-processing procedures lead to different accuracy ratios.

- To provide a generic procedure for pre-processing.

\subsection{Experiment guidelines}

Different pre-processing procedures will be applied to the original Data Set $\left(\mathrm{DS}_{0}\right)$. Thus, a group of different pre-processed Data Sets will be obtained (DS $)_{\mathrm{i}}$. Each of these Data Sets will be subjected to a classification tree for a total of 10 times in order to calculate (by Cross Validation technique) the precision provided by the tree in each Data Set.

Assuming that the trees will be generated with the same criteria for construction, expansion and pruning, the differences between the accuracies achieved will only be attributable to the input Data Set in each case and they will depend entirely on the pre-processing which they have undergone.

Thus, it is possible to empirically establish how different pre-processing procedures lead to different levels of accuracy under the same predictive model. Figure 2 shows a general schema for the accuracy comparison, depending on the input Data Sets.

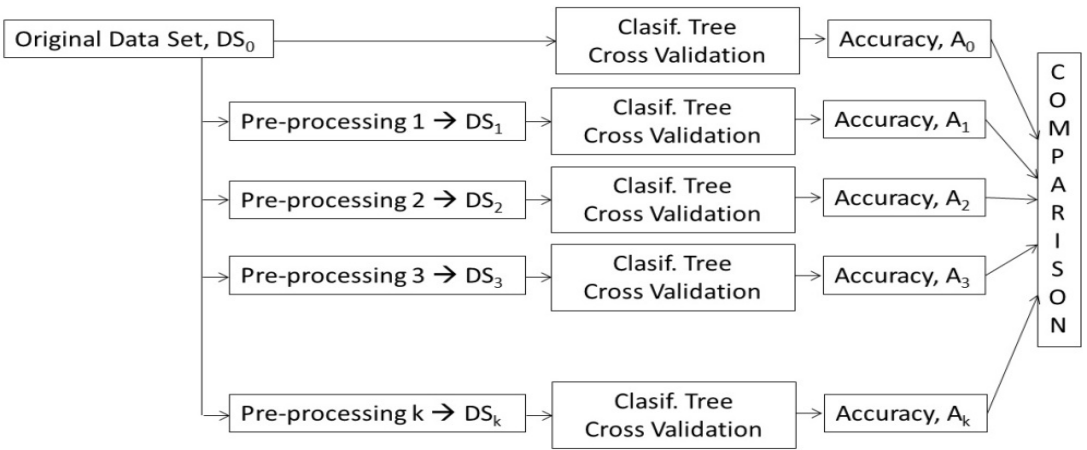

Figure 2: Accuracy comparison schema depending on different input Data Sets. 
Both pre-processing and classification tree constructions are implemented using WEKA [3], the most extended Data Mining open access tool in academic frameworks.

\section{Original Data Set description}

Guvenir et al. [4] proposed an accurate method (the VF15 algorithm) for classification tasks, tested with their own cardiac data base. The original Data Set is available at UCI Machine Learning Repository [5]. The aim of their study was to classify the type of cardiac arrhythmia (there are 16 types for such a class variable). The original Data Set consists of 279 features (attributes or columns): Patients' personal data and the values registered by the electrocardiogram. Most of them (206) are linear attributes and the rest are nominal. There are several missing values. The Data Set consists of 452 patient records (rows or instances).

In this paper the same original Data Set (further $\mathrm{DS}_{0}$ ) is used as input data, and is subjected to a classification tree. The achieved accuracy $\left(\mathrm{A}_{0}\right)$ will be considered as accuracy of reference.

Figure 3 shows the head of $\mathrm{DS}_{0}$, the first 15 attributes (from 280) and the first 30 records (from 452), where missing values are denoted by '?'.

\begin{tabular}{|c|c|c|c|c|c|c|c|c|c|c|c|c|c|c|c|c|c|c|c|c|c|c|c|c|c|c|c|c|c|}
\hline 1 & $f 2$ & f3 & $f 4$ & 5 & $f 6$ & f7 & f8 & $f 9$ & 10 & 11 & 2 & 3 & 14 & 5 & 6 & & 8 & 9 & $f 20$ & & 22 & 23 & 24 & & $f 26$ & $f$ & & & \\
\hline 75 & 0 & 90 & 80 & 91 & 193 & 3 & 174 & 121 & -1 & 13 & 64 & -2 & ? & 63 & 0 & 2 & 4 & 0 & 0 & 32 & 0 & 0 & 0 & 0 & 0 & 0 & 0 & 14 & 20 \\
\hline 56 & 1 & 165 & 6 & 81 & $t$ & 4 & 149 & 39 & 25 & 37 & -1 & 3 & ? & 53 & 0 & 48 & 0 & 0 & 0 & 24 & 0 & 0 & 0 & 0 & 0 & 0 & 0 & 64 & 0 \\
\hline & 0 & 77 & 95 & 8 & 3 & 3 & 1 & 10 & 96 & 34 & 70 & 66 & 23 & 75 & 0 & 40 & 8 & 0 & 0 & 24 & 0 & 0 & 0 & 0 & 0 & 0 & 0 & 56 & 52 \\
\hline & 0 & 75 & 94 & 100 & 2 & 3 & & 1 & 28 & 11 & 5 & 2 & & 7 & 0 & 72 & 20 & 0 & 0 & 48 & 0 & 0 & 0 & 0 & 0 & 0 & 0 & 54 & 36 \\
\hline & 0 & 0 & 8 & 88 & 181 & 3 & & 103 & -16 & 13 & 61 & 3 & : & : & 0 & 48 & 40 & 0 & 0 & 28 & 0 & 0 & 0 & 0 & 0 & 0 & 0 & 40 & 24 \\
\hline & 0 & 169 & 51 & 100 & 167 & 321 & 174 & 91 & 107 & 66 & 52 & 88 & : & 8 & 0 & 3 & 48 & 0 & 0 & 20 & 0 & 0 & 0 & 0 & 0 & 0 & 0 & 44 & 36 \\
\hline 0 & 1 & 160 & 52 & 77 & 129 & 377 & 133 & 77 & 7 & 49 & 75 & 65 & ? & 70 & 0 & 4 & 0 & 0 & 0 & 24 & 0 & 0 & 0 & 0 & 0 & 0 & 0 & 40 & 32 \\
\hline 49 & 1 & 62 & 54 & 78 & 0 & 376 & 157 & 70 & 67 & 7 & 8 & 51 & ? & 67 & 0 & 44 & 36 & 0 & 0 & 24 & 0 & 0 & 0 & 0 & 0 & 0 & 0 & 52 & 32 \\
\hline 44 & 0 & 68 & 56 & 84 & 118 & 3 & 1 & 63 & 61 & 69 & 7 & 66 & 84 & 64 & 0 & 40 & 0 & 0 & 0 & 2 & 0 & 0 & 0 & 0 & 0 & 0 & 0 & 44 & 12 \\
\hline 50 & 1 & 167 & 6 & 89 & 130 & 3 & 1 & 7 & 8 & 34 & 70 & 71 & ? & 63 & 0 & 4 & 40 & 0 & 0 & 28 & 0 & 0 & 0 & 0 & 0 & 0 & 0 & 66 & 24 \\
\hline 62 & 0 & 170 & 72 & 1 & 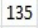 & & & 83 & 72 & 71 & & 72 & ? & 70 & 2 & 36 & 48 & & & & 0 & & & 0 & 0 & 0 & 0 & 52 & 0 \\
\hline 45 & 1 & 165 & 8 & 77 & 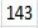 & & & 65 & 12 & 37 & & 26 & i & & 0 & 7 & 28 & 0 & 0 & & 0 & 0 & 0 & . & 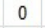 & 0 & 0 & O & 20 \\
\hline 54 & 1 & 72 & 58 & 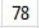 & . & 3 & 1 & 81 & -24 & 42 & 41 & -1 & : & I & 0 & 72 & 0 & 0 & 0 & 24 & 0 & 0 & 0 & 0 & 0 & 0 & 0 & 4 & 44 \\
\hline 30 & 0 & 170 & 73 & 91 & 180 & 3 & 1 & 104 & 68 & 5 & 60 & 6 & $?$ & 5 & 0 & 92 & 0 & 0 & 0 & 32 & 0 & 0 & 0 & 0 & 0 & 0 & 8 & 48 & 20 \\
\hline 44 & 1 & 160 & 88 & 77 & 158 & 399 & 163 & 94 & 46 & 20 & 45 & 40 & ? & 72 & 0 & 80 & 0 & 0 & 0 & 28 & 0 & 0 & 0 & 0 & 0 & 0 & 20 & 72 & 0 \\
\hline
\end{tabular}

Figure 3: $\quad$ Extract of the original Data Set, $\mathrm{DS}_{0}$.

\section{Classification tree models and accuracy measurement}

As the Data Sets pre-processing designs presented in section 4 are based on the accuracy achieved after being processed by the J48 classification tree, it is mandatory to present these concepts previously. Thus, in this section we introduce some classification tree concepts and the accuracy measure that will be used for each preprocessed Data Set, $\mathrm{DS}_{\mathrm{i}}$.

\subsection{Classification trees concepts}

C4.5, presented by Quinnlan [6] is probably one of the most extended for solving forecasting real problems in Medicine: Block et al. [7] presented a comparative 
study of forecasting methods in Medicine, where C4.5 was pointed as a very accurate and suitable algorithm. C4.5 has been widely used, for example to predict the posology (Chan et al. [8]), or even for early cancer diagnosis (e.g. Polat et al. [9] and Takir and Bouridane [10] among others). C4.5 was first presented as a significant improvement to ID3, which only managed categorical attributes. C4.5 generates a Classification Tree with the input data sets by boosting at each node, based on information gain. Leafs of the tree correspond to the class variable instances. Each branch (from root to leaf) is interpreted as a classification rule.

WEKA provides an accurate Java implementation of C4.5: the J48 Classification Tree.

\subsection{Accuracy measurement}

In this paper, a Cross Validation methodology is used for measuring the classification accuracy reached by applying the J48 algorithm over each Data Set. Accuracy, $\mathrm{A}_{\mathrm{i}}$, associated to each Data Set, $\mathrm{DS}_{\mathrm{i}}$ is assumed to be the average of ten executions of the J48 algorithm. Figure 4 shows $A_{0}=64.3805$ after applying Cross Validation over $\mathrm{DS}_{0}$ classification.

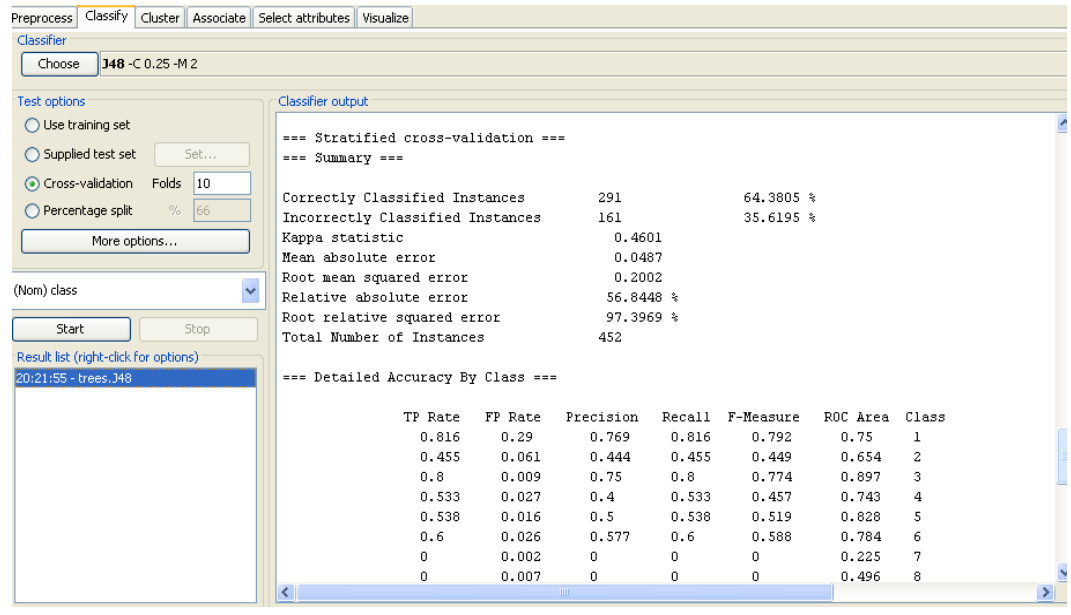

Figure 4: WEKA's cross validation over $\mathrm{DS}_{0}$.

\section{Generating different data sets from ad-hoc pre-processing}

The original Data Set, $\mathrm{DS}_{0}$, inputs into the J48 Classification Tree, and after a Cross Validation procedure, provides an accuracy ratio of reference $\mathrm{A}_{0}=64.38$.

Next, $\mathrm{DS}_{0}$ is subjected to different pre-processing routines under a trial and error methodology, in order to improve (or "approximately" maintain) the best accuracy ratio, $A_{i}$, achieved in previous steps. 
First, the missing value detection notes that attribute f14 have an $83 \%$ missing value ratio. The rest of the columns have an acceptable missing value ratio (less than $50 \%)$. By deleting $\mathrm{f1} 14$ attribute, we obtain $\mathrm{DS}_{1} \cdot \mathrm{A}_{1}=64.16\left(\approx \mathrm{A}_{0}\right)$. This is a very similar accuracy and it has one column less, so both $\mathrm{DS}_{0}$ and $\mathrm{DS}_{1}$ will be considered. For the next pre-processing variations, we focus on $\mathrm{DS}_{1}$ (Figure 5).

Looking for outliers and extreme values, WEKA finds a set of attributes that could be avoided from $\mathrm{DS}_{1}$, because there is a $10 \%$ outlier and extreme value ratio. So, by different parameterizing of outliers and extreme boundaries, we obtain $\mathrm{DS}_{2}$ and $\mathrm{DS}_{3}$, with $\mathrm{A}_{2}=64.38\left(=\mathrm{A}_{0}\right)$ and $\mathrm{A}_{3}=61.95\left(<\mathrm{A}_{0}\right)$, respectively. $\mathrm{DS}_{2}$ achieves the same accuracy level as $\mathrm{DS}_{0}$ (with 15 attributes less), so $\mathrm{DS}_{2}$ will be focused for the next pre-processing variations.

Sometimes the missing value replacement could improve the sample quality, so missing values on $\mathrm{DS}_{2}$ are replaced with their corresponding attribute average value. This provides $\mathrm{DS}_{4}$, with worse accuracy levels $\left(\mathrm{A}_{4}<\mathrm{A}_{0}\right)$. In fact, there is no sense in replacing missing ECG values for other patients. Even by including f14 (with $83 \%$ missing) in $\mathrm{DS}_{2}$ to obtain $\mathrm{DS}_{5}$, the accuracy does not improve $\left(\mathrm{A}_{5}=64.38\right)$.

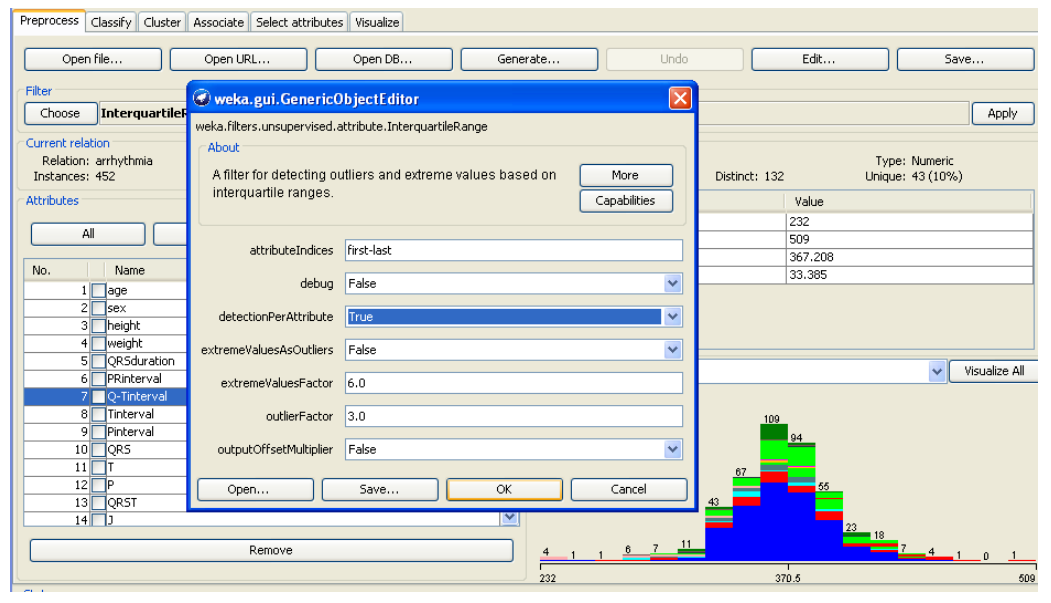

Figure 5: $\quad$ Outlier detection over $\mathrm{DS}_{0}$

This study faces a very broad problem (280 attributes per 452 records). If accuracy was maintained, a reduced set of attributes would be preferable because it would generate reduced classification rule sets. Thus, it seems to be necessary to select the most important attributes. Automatic feature selection over $\mathrm{DS}_{0}$ and $\mathrm{DS}_{2}$ provides $\mathrm{DS}_{6}$ and $\mathrm{DS}_{7}$, respectively.

Also, after applying automatic discretization $\mathrm{DS}_{9}$ and $\mathrm{DS}_{10}$ were created (over $\mathrm{DS}_{0}$ and $\mathrm{DS}_{6}$ respectively: the Data Sets with best accuracies at the moment), both Data Sets were processed by the Classification Tree with $A_{9}=64.38$ and $\mathrm{A}_{10}=64.40$. Neither improves $\mathrm{A}_{6}$ accuracy. 
Just as a test, the authors combined some of the Data Sets to increase accuracy, by considering extra attributes, removed in previous experiments. For example, defining $\mathrm{DS}_{8}$ as the union of $\mathrm{DS}_{6}$ and $\mathrm{DS}_{7}$, the experiment equals the best accuracies obtained so far $\left(\mathrm{A}_{6}=68.36\right)$ but considering larger Data Sets with still too many attributes. Besides, although this case requires numerical data to be treated as numbers (and not as categorical), the authors tried to apply the WEKA's automatic discretization over the best Data Sets, $\mathrm{DS}_{0}$ and $\mathrm{DS}_{6}$, and neither improves accuracy.

Next, Table 1 summarizes the previously described pre-processing actions, and their respective accuracy levels.

Table 1: $\quad$ Pre-processing actions.

\begin{tabular}{|c|c|c|c|}
\hline$D S$ & Pre-processing routine & Results & Accur. \\
\hline $\mathrm{DS}_{0}$ & No pre-processed & $\mathrm{DS}_{0}$ & 64.38 \\
\hline $\mathrm{DS}_{1}$ & $\begin{array}{l}\text { Missing values }>50 \% \\
\text { detection and attribute } \\
\text { filtering. }\end{array}$ & $\begin{array}{l}\mathrm{DS}_{0}-\{\mathrm{f} 14\} \\
\text { f14 has } 83 \% \text { missing }\end{array}$ & 64.16 \\
\hline $\mathrm{DS}_{2}$ & $\begin{array}{l}\text { Outliers (3) and } \\
\text { Extreme Values (6) } \\
\text { Detection }>10 \% \\
\end{array}$ & $\begin{array}{l}\mathrm{DS}_{1}-\{\mathrm{f} 27, \mathrm{f} 53, \mathrm{f} 75, \mathrm{f} 87, \mathrm{f} 90, \mathrm{f} 123, \mathrm{f} 135 \\
\mathrm{f} 170, \mathrm{f} 192, \mathrm{f} 210, \mathrm{f} 220, \mathrm{f} 223, \mathrm{f} 250, \mathrm{f} 260\}\end{array}$ & 64.38 \\
\hline $\mathrm{DS}_{3}$ & $\begin{array}{l}\text { Outliers }(1,5) \text { and } \\
\text { Extreme Values (3) } \\
\text { Detection }>10 \%\end{array}$ & $\begin{array}{l}\mathrm{DS}_{1}-\{\mathrm{f} 27, \mathrm{f} 51, \mathrm{f} 53, \mathrm{f} 75, \mathrm{f} 87, \mathrm{f} 88, \mathrm{f} 90, \\
\text { f101, f123, f135, f148, f170, f180, f192, } \\
\text { f210, f220, f223, f250, f260\} }\end{array}$ & 61.95 \\
\hline $\mathrm{DS}_{4}$ & $\begin{array}{l}\text { Missing values } \\
\text { completed with average } \\
\text { values }\end{array}$ & $\begin{array}{l}\mathrm{DS}_{2} \text { with average missing values } \\
\text { completed }\end{array}$ & 62.61 \\
\hline $\mathrm{DS}_{5}$ & $\begin{array}{l}\text { Recovering original } \\
\text { missing values }\end{array}$ & $\begin{array}{l}\mathrm{DS}_{2} \text { with original missing value } \\
\mathrm{DS}_{2}+\{\mathrm{f} 14\}\end{array}$ & 64.38 \\
\hline $\mathrm{DS}_{6}$ & $\begin{array}{l}\text { Automatic Feature } \\
\text { Selection over } \mathrm{DS}_{0}\end{array}$ & $\begin{array}{l}\text { \{f5, f7, f8,f11, f15, f40, f76, f90, f93, } \\
\text { f100, f103, f112, f114, f121, f190, f197, } \\
\text { f211, f217, f222, f224, f228, f247, f248, } \\
\text { f267, f277, f279\} }\end{array}$ & 68.36 \\
\hline $\mathrm{DS}_{7}$ & $\begin{array}{l}\text { Automatic Feature } \\
\text { Selection over } \mathrm{DS}_{2}\end{array}$ & $\begin{array}{l}\text { \{f5, f7, f8, f11, f14, f38, f54, f66, f85, } \\
\text { f87, f94, f97, f106, f108, f115, f181, } \\
\text { f187, f206, f210, f215, f234, f252, f262, } \\
\text { f264\} }\end{array}$ & 63.94 \\
\hline$D S_{8}$ & $\begin{array}{l}\text { Automatic Feature } \\
\text { Selection best union }\end{array}$ & $D S_{6} \cup D S_{7}$ & 68.36 \\
\hline$D S_{9}$ & $\begin{array}{l}\text { Automatic discretization } \\
\text { over } \mathrm{DS}_{0}\end{array}$ & $D S_{0}$ : all attributes are discrete & 64.38 \\
\hline$D S_{10}$ & $\begin{array}{l}\text { Automatic discretization } \\
\text { over } \mathrm{DS}_{6}\end{array}$ & $D S_{6}:$ all attributes are discrete & 60.40 \\
\hline
\end{tabular}

Figure 6 shows the pre-processing experiment trace. 


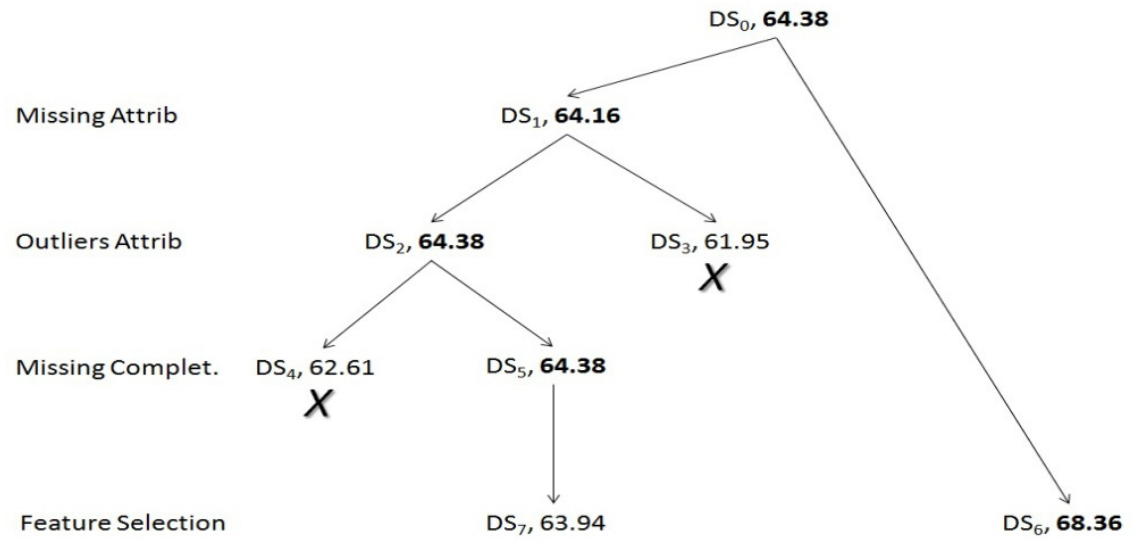

Figure 6: $\quad$ Pre-processing experiment trace.

\section{Conclusions}

\subsection{An adequate pre-processing improves the final accuracy}

- The accuracy initially reached $\left(\mathrm{A}_{0}=64.38\right)$ by processing the original Data Set with the J48 classification tree is significantly improved $\left(A_{6}=68.36\right)$, if the same technique (equally parameterized) is applied to a well pre-processed Data Set.

- Missing values must be detected and if they appear very often at the same attribute, they must be removed. However, depending on the problem context, the missing values replacement is not suitable

- Some values, statistically considered as outliers, must be maintained in the Data Set. They may indicate certain pathologies. If wrong attributes are removed (even containing outliers), the final accuracy could worsen. So, $A_{3}$ is 2 percentage points worse than $\mathrm{A}_{0}$.

- Where the amount of records is too small in comparison to the number of attributes, it is preferable not to delete any row and efforts must focus on the attribute selection.

- If numerical data could be discretized without loss of critical information, it could lead to much more precise forecasting, but it must depend on the expert's criteria. In this case, the numerical attributes must be treated as numbers.

\subsection{Generic procedure for pre-processing}

The authors propose a fuzzy-greedy accuracy improving procedure under a trial and error methodology that progressively improves (or "approximately" maintains) the best accuracy ratio, $\mathrm{A}_{\mathrm{i}}$, as achieved in previous steps.

Some questions about the missing value treatment and replacement, the suitability of discretization, removing outliers and others must be considered. 
Figure 7 shows a generic schema of such a procedure. The main questions are ordered and points are given about how to deal with attributes or records.

1) Missing Values $\left\{\begin{array}{r}\begin{array}{r}\text { Records: If enought, REMOVE } \\ \text { Else, ASK EXPERT FOR REPLACEMENT }\end{array} \\ \text { Attributes: If too many, REMOVE }\end{array}\right.$
2) Outliers

3) Discretization

ASK EXPERTFOR POSSIBLE DISCRETIZATION AND CRITERIA

4) Feature Selection Attributes: If too many, SELECT BEST CORRELATED

Figure 7: Generic procedure for pre-processing.

\section{Acknowledgement}

This study is part of the Bancaja-UMH project: "Minería de Datos sobre Análisis Preoperatorios para Servicios Hospitalarios Quirúrgicos y de Anestesia" (Data Mining with Presurgery Tests for Surgery and Anaesthesiology Hospital Departments).

\section{References}

[1] Chen, Z. Data Mining and Uncertaining Reasoning. An Integrated Approach. Wiley Interscience, 2001

[2] Berry, M.J. and Linoff, G. Mastering Data Mining. The Art and Science of Customer Relationship Management. Wiley, 2000

[3] Machine Learning Group at the University of Waikato, New Zeland. www.cs.waikato.ac.nz/ml/weka/

[4] Guvenir, H.A., Acar, B., Demiroz, G. and Cekin, A. A Supervised Machine Learning Algorithm for Arrhythmia Analysis. Proc. of the Computers in Cardiology Conference, Lund, Sweden, 1997

[5] Machine Learning Repository, University of California, Irvine, USA. archive.ics.uci.edu/ml/

[6] Quinlan, J. R. C4.5: Programs for Machine Learning. Morgan Kaufmann Publishers, 1993

[7] Block, P., Paern, J., Hüllermeier, E., Sanschagrin, P., Sotriffer, C. and Klebe, G. Physicochemical Descriptors To Discriminate Protein-Protein Interactions In Permanent And Transient Complexes Selected By Means Of Machine Learning Algorithms. Wiley Inter Science, Proteins: Structure, Function, and Bioinformatics 65, pp. 607-622, 2006 
[8] Chan, A.L., Chen, J.X. and Wang, H.Y. Application of Data Mining to Predict the Dosage of Vancomycin as an Outcome Variable in a Teaching Hospital Population. Dustri-Verlag. International Journal of Clinical Pharmacology and Therapeutics 44 (11), pp-533-538, 2006

[9] Polat, K., Sahan, S., Kodaz, H. and Gunes, S. A New Classification Method For Breast Cancer Diagnosis: Feature Selection Artificial Immune Recognition System (FS-AIRS). Springer-Verlag. Advances in Natural Computation 2, Proc. Lecture Notes in Computer Science 3611, pp. 830838, 2005

[10] Tahir, M.A. and Bouridane, A. Novel Round-Robin Tabu Search Algorithm For Prostate Cancer Classification And Diagnosis Using Multispectral Imagery. IEEE-Inst. Electrical Electronics Eng. IEEE Transactions on Information Technology in Biomedicine 10 (4), pp. 782-793, 2006 